\title{
Determination of the carbapenem resistance in Escherichia coli isolated from samples obtained from Shahrekord hospitals and determination of their minimum inhibitory concentration
}

\author{
Farshad Kakian $^{\mathbb{(}}$, Behnam Zamzad ${ }^{2}{ }^{(\mathbb{D}}$, Abolfazl Gholipour ${ }^{(\mathbb{D}}$, Kiarash Zamanzad ${ }^{3}$ \\ 'Department of Bacteriology \& Virology, School of Medicine, Shiraz University of Medical Sciences, Shiraz, Iran \\ ${ }^{2}$ Department of Medical Bacteriology, Shahrekord University of Medical Sciences, Shahrekord, Iran \\ ${ }^{3}$ Student of Veterinary Medicine, University of Shahrekord, Shahrekord, Iran
}

*Corresponding Author: Behnam Zamzad, Cellular and Molecular Research Center, Shahrekord University of Medical Sciences, Shahrekord, Iran, Cell phone No: +98913 181 5136, Email: bzamanzad@yahoo.com

\begin{abstract}
Background and aims: Carbapenems are the final-line treatments for multidrug-resistant, gram-negative infections. The patterns of resistance to carbapenems among hospital bacterial pathogens vary widely across different hospitals in a country. Considering that Escherichia coli is one of the most important causes of nosocomial infections, it is essential to study its drug resistance.

Methods: In this descriptive-analytical study, a total of 80 samples of $E$. coli isolated from inpatients with urinary tract infections (UTIs) were collected in different wards (i.e., women, urology, infectious, and ICU) of Shahrekord hospitals. After the diagnosis and confirmation of bacteria by standard bacteriological methods, their sensitivity to imipenem and meropenem was investigated by the antibiogram (diskdiffusion) method. Then, the minimum inhibitory concentration (MIC) was determined by the E-test strip according to the Clinical and Laboratory Standards Institute (CLSI) standard.

Results: In this study, resistance to meropenem and imipenem by antibiogram (disc diffusion) was observed in 21 (25.26\%) and 20 (25\%) of the isolates, respectively. Twenty isolates had MIC $\geq 4 \mu \mathrm{g} / \mathrm{mL}$ for meropenem, 13 isolates demonstrated $\mathrm{MIC} \geq 4 \mu \mathrm{g} / \mathrm{mL}$ for imipenem, and 14 isolates had $1 \leq \mathrm{MIC}<4 \mu \mathrm{g} / \mathrm{mL}$ and were semi-sensitive.

Conclusion: In general, E. coli had significant resistance to carbapenems. Therefore, rapid and accurate identification of these strains can be a major step to the treatment and control of these strains and prevention of the spread of the resistance.

Keywords: Escherichia coli, Carbapenems, Meropenem, Imipenem, Minimum inhibitory concentration
\end{abstract}

Received: 14 October 2018, Accepted: 27 June 2019, ePublished: 31 December 2019

\section{Introduction}

Microorganisms causing nosocomial infections lead to severe problems for patients and the health system due to antibiotic resistance (1). In some countries, the rate of nosocomial infections is high due to the lack of monitoring, the lack of infection prevention, the inappropriate use of antibiotics, and excessive overcrowding in the hospitals (2). According to Yang et al, the Enterobacteriaceae family, particularly Escherichia coli, is responsible for various types of infections, especially urinary tract infections (UTIs). These infections account for $30 \%-40 \%$ of nosocomial infections and are the most common cause of gram-negative septicemia in inpatients $(4,5)$. The most common etiologic factor for UTI is E. coli, which accounts for most of these cases (6,7). Carbapenems include ertapenem, imipenem, doripenem, and meropenem (8), which are nowadays regarded as selective drugs that are used to treat serious infections in multidrug-resistant Enterobacteriaceae and produce extended-spectrum $\beta$-lactamases $(9,10)$. In fact, they are the final-line antibiotics for the treatment of multidrug resistance (11). In addition, carbapenems are considered as selective therapeutic agents for gramnegative, penicillin- and cephalosporin-resistant bacterial infections due to their extended-spectrum and the lack of hydrolysis by $\beta$-lactamases enzymes (8). The excessive increase in antibiotic resistance among gramnegative bacteria has challenged drug therapies $(12,13)$. In this study, the frequency of $E$. coli strains resistant to meropenem and imipenem was investigated by the disk diffusion method, followed by determining their minimum inhibitory concentrations (MICs) by using the E-test strip. Clinicians can select appropriate antibiotics to rapidly eradicate the infections caused by carbapenems by obtaining information on the prevalence and rate of

(C) 2019 The Author(s); Published by Shahrekord University of Medical Sciences. This is an open-access article distributed under the terms of the Creative Commons Attribution License (http://creativecommons.org/licenses/by/4.0), which permits unrestricted use, distribution, and reproduction in any medium, provided the original work is properly cited. 
resistance to these bacteria in $E$. coli isolates from UTI samples in different regions of Iran and across the world. It also avoids a waste of time, the futile consumption of drugs, or even an increase in resistance to the mentioned bacteria.

\section{Materials and Methods}

In this study, $80 \mathrm{E}$. coli isolates were collected from UTI inpatients in different wards of the hospitals (e.g., urology, obstetrics and gynecology, internal medicine, and ICU) in Shahrekord during April to October, 2016. The inpatients had acquired UTI 48-72 hours after admission and their first urinary culture was negative. The study protocol was approved by Shahrekord University of Medical Sciences under the code IR.SKUMS.REC.1394.282. First, the samples were cultured on blood agar and eosin methylene blue (HiMedia Company, India) and then identified by biochemical differential tests such as triple sugar iron agar, Simon citrate, methyl red/Voges-Proskauer, indole motility, oxidase and catalase. After the identification and phenotypic confirmation of $E$. coli, the colony of pure bacteria was stored for subsequent tests. For this purpose, the pure colony was inoculated in 1.5 microtubes containing 700 $\mu \mathrm{L}$ of sterilized TSB culture medium and incubated at $37^{\circ} \mathrm{C}$ overnight. After the growth, $300 \mu \mathrm{L}$ of sterilized glycerol was added to it and stored under $-70^{\circ} \mathrm{C}$ in the freezer. To determine the antibiotic resistance, a suspension of pure bacteria equivalent to the $0.5 \mathrm{McFarland}$ was prepared and then passaged on the surface of Mueller-Hinton agar medium and incubated at $37^{\circ} \mathrm{C}$ for $18-24$ hours. Antibiotic susceptibility was determined by the disk diffusion (KirbyBauer) method for two antibiotics, namely, meropenem $(10 \mu \mathrm{g})$ and imipenem $(10 \mu \mathrm{g})$ obtained from MAST Company, UK. After the incubation, the diameter of the growth inhibition zone was measured and interpreted in accordance with the CLSI Table. In addition, E. coli ATCC 25922 was used for the quality control of susceptibility testing. MIC was determined by using the E-test strips (Liofilchem, Italy) at a concentration ranging from 0.002 to $32 \mathrm{mg} / \mathrm{L}$. Next, bacterial suspension was prepared from pure cultures to match the turbidity of the $0.5 \mathrm{McF}$ arland and cultured on the Mueller-Hinton agar. Then, the strip was placed on the medium and incubated at $37^{\circ} \mathrm{C}$ for 16-18 hours. The antibiotic release from the strip into the surrounding area formed an oval zone and the MIC extended to where it crossed the strip (14-18).

\section{Results}

The clinical samples of inpatients with UTI in different wards of Hajar and Kashani hospitals in Shahrekord were isolated, the details of which are presented in Table 1. As shown in Table 1, most isolates were obtained from the inpatients in the urology department. According to the results of the disk diffusion method, 21 and 20 isolates of $E$. coli were resistant to meropenem and imipenem, respectively, and 5 isolates had intermediate resistance (semi-sensitive).

The highest number of resistant isolates of $E$. coli to meropenem and imipenem was isolated from the urology department, and none of the isolated strains from the internal medicine department had MIC $\geq 4$ for meropenem. The E-test strips were read from the top (high concentrations) to the bottom (low concentrations of the antibiotic) after 18 hours of incubation (Figure 1 and 2). According to the E-test results, 20 isolates were resistant to meropenem and 13 isolates to imipenem (MIC $\geq 4$ ), indicating the high accuracy of the E-test method compared to the disk diffusion method for detecting resistant and semi-sensitive strains. The frequency of carbapenemresistant and sensitive isolates is presented in Table 2.

\section{Discussion}

Opportunistic bacteria are the cause of nosocomial infections in different hospital wards and cause certain infections such as pneumonia, UTIs, and bacteremia after hospitalization. The underlying factors such as surgery, the weakness of the inpatient's immune system, and longterm hospital stay contribute to the acquisition of these infections. Further, E. coli, as the most common cause of UTIs, is considered as an important pathogen in the development of neonatal meningitis, respiratory infections, sepsis, fever and frequent urination, and disseminated intravascular coagulation in the inpatients in different wards of the hospital. Furthermore, antibiotic resistance is very common among the $E$. coli strains, which is due to the acquisition of multiple resistance factors. Today, bacterial resistance to various antibiotics has become a global problem and the uncontrolled administration of antibiotics for the treatment of bacterial infections has led to the selection

Table 1. The frequency distribution of Escherichia coli isolated from clinical ward

\begin{tabular}{lccc}
\hline Clinical Ward & $\begin{array}{c}\text { E. coli } \\
(\text { No. })\end{array}$ & $\begin{array}{c}\text { Resistant to } \\
\text { Meropenem (MIC } \geq 4)\end{array}$ & $\begin{array}{c}\text { Resistant to } \\
\text { Imipenem (MIC } \geq 4)\end{array}$ \\
\hline $\begin{array}{l}\text { Urology } \\
\begin{array}{l}\text { Gynecology } \\
\text { and obstetrics }\end{array}\end{array}$ & 16 & 10 & 7 \\
$\begin{array}{l}\text { Infection } \\
\text { Internal }\end{array}$ & 14 & 5 & 2 \\
$\begin{array}{l}\text { Intensive care } \\
\text { unit }\end{array}$ & 5 & 0 & 1 \\
\hline
\end{tabular}

Table 2. The frequencies of strains according to the minimum inhibitory concentrations of imipenem and meropenem

\begin{tabular}{lccc}
\hline Antibiotics & $\begin{array}{c}\mathbf{R} \\
(\mathbf{M I C} \geq \mathbf{4})\end{array}$ & $\begin{array}{c}\mathbf{I} \\
(\mathbf{4}>\mathbf{M I C} \geq \mathbf{1})\end{array}$ & $\begin{array}{c}\mathbf{S} \\
(\mathbf{M I C}<\mathbf{1})\end{array}$ \\
\hline Meropenem & 20 & 8 & 52 \\
Imipenem & 13 & 6 & 61 \\
\hline
\end{tabular}

Note. MIC: minimum inhibitory concentration. 


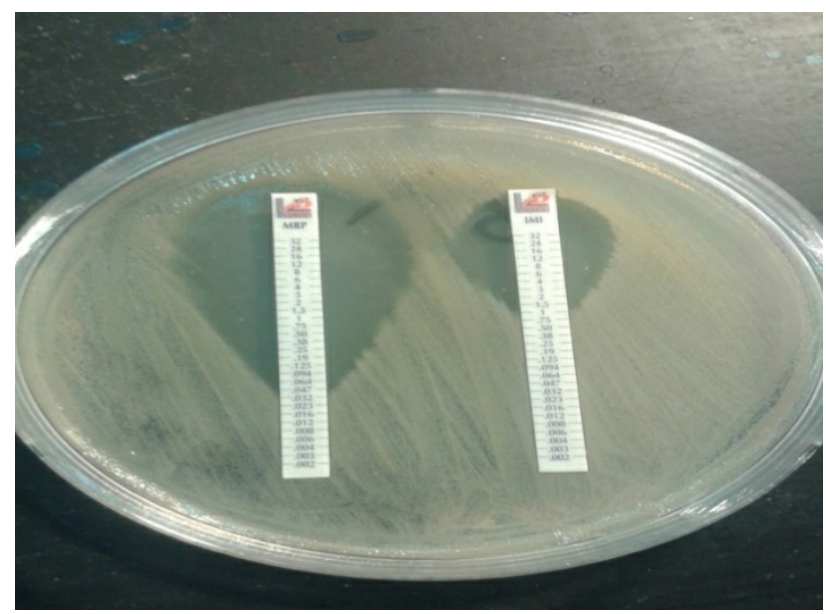

Figure $\mathbf{1}$. The E-test strips of meropenem and imipenem for meropenem and imipenem-susceptible isolate (MIC $<1$ ).

of resistant strains (19). Antibiotic resistance patterns among nosocomial pathogenic bacteria may significantly vary from one country to another or in different regions of a country (20). Moreover, most pathogens are relatively resistant to some of the new antibiotics such as extendedspectrum cephalosporins (e.g., cefotaxime and ceftazidime). Previously, imipenem was considered the most active drug for the infections. Recently, however, evidence demonstrates the spread of imipenem-resistant strains $(21,22)$. The wide emergence of resistance to imipenem and meropenem is a serious threat to future treatment. Additionally, antibiotic resistance and susceptibility vary from country to country due to environmental factors and the use of different antimicrobial agents (23). Previous research has also confirmed the selection of the E-test method as a precise and sensitive method for determining the susceptibility of bacteria to antibiotics (24). In a similar study conducted by Nobari et al regarding the investigation of resistance to carbapenems on 180 isolates, it was revealed that 42, 29, and 14 isolates were resistant to meropenem, ertapenem, and imipenem, respectively (25). Therefore, resistance to E. coli appears to be constantly changing and it is essential to determine this change in different regions. In a study by Simhon et al, the sensitivity of imipenem decreased from $98 \%$ in 1990 to $64.1 \%$ in 2000 and the ciprofloxacin sensitivity reduced from $50.5 \%$ to $13 \%$ (26). Additionally, Bora et al. investigated resistance to carbapenems in E. coli and Klebsiella pneumoniae strains by using phenotypic tests and found that 41 of $216 \mathrm{E}$. coli isolates and 39 of 185 $K$. pneumoniae isolates were resistant to carbapenems (27). Similarly, in their study in Al-Zahra hospital of Isfahan between the time period of March 2012 and December 2012, Moayednia et al reported that 9, 14, and 8 out of 720 E. coli isolates were resistant to meropenem, imipenem, and ertapenem, respectively, showing a lower resistance rate to meropenem and imipenem in the past compared to our current study (28). In a study conducted by Shahcheraghi

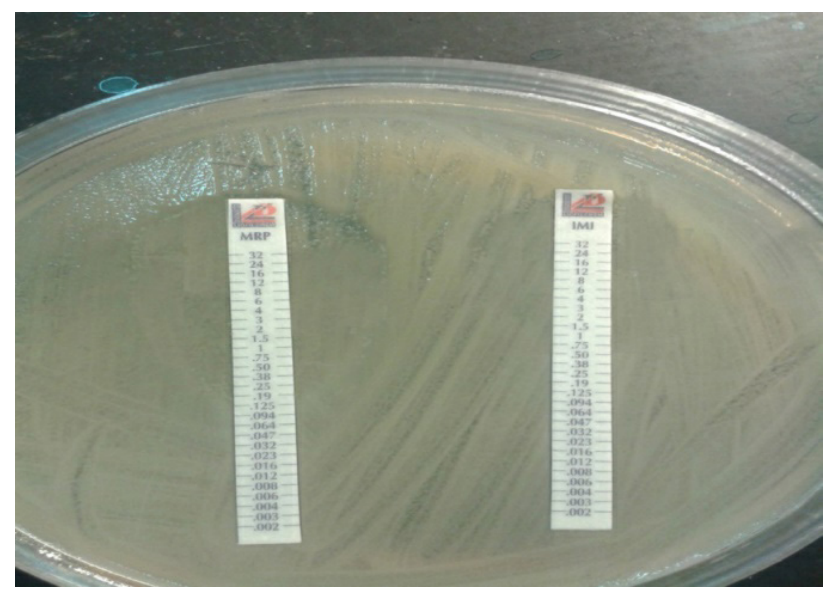

Figure 2. The E-test strips for meropenem and imipenem-resistant isolate $(\mathrm{MIC} \geq 4)$

et al in Tehran, 9 out of 244 isolates of $E$. coli were identified as meropenem-resistant, 1 isolate as imipenem-resistant, and 2 isolates as ertapenem-resistant (29). In addition, a study in a hospital in northern Palestine by Adwan et al on 79 isolates of $E$. coli showed that 30 and 35 isolates were resistant to meropenem and imipenem, respectively, which can represent an increased resistance to another region of the world (30). This is also consistent with the results of a study by Kanchanadevi and Sekaran in which 25 out of 76 E. coli isolates, 2 out of 9 Klebsiella isolates, and 42 out of 60 Pseudomonas isolates were resistant to imipenem according to the E-test (31). Resistance to carbapenems is very important because if the isolates from nosocomial infections are resistant to carbapenems, they will be also resistant to other antibiotics. Therefore, other antibiotics such as carbapenem-Sulbactam, colistin, or tigecycline should be used to treat these types of infections. Studying the resistance rate of $E$. coli isolates will provide adequate information for physicians to develop appropriate methods for the treatment of the infections due to this bacterium (2).

\section{Conclusion}

This study indicates a significant prevalence of resistance to the mentioned antibiotics, and this could be an alarm signal for the Healthcare.

Conflict of Interests

None.

\section{Acknowledgments}

This study was a research project approved by Shahrekord University of Medical Sciences (approval No. 2057) on 6 March 2016. We, hereby, gratefully thank all the people who assisted us in conducting this work.

\section{References}

1. Curtis LT. Prevention of hospital-acquired infections: review of non-pharmacological interventions. J Hosp Infect. 
2008;69(3):204-19. doi: 10.1016/j.jhin.2008.03.018

2. Lagamayo EN. Antimicrobial resistance in major pathogens of hospital-acquired pneumonia in Asian countries. Am J Infect Control. 2008;36(4 Suppl):S101-8. doi: 10.1016/j. ajic.2007.10.020.

3. Yang J, Ye L, Wang W, Luo Y, Zhang Y, Han L. Diverse prevalence of $16 \mathrm{~S}$ rRNA methylase genes armA and rmtB amongst clinical multidrug-resistant Escherichia coli and Klebsiella pneumoniae isolates. Int J Antimicrob Agents. 2011;38(4):348-51. doi: 10.1016/j.ijantimicag.2011.04.021.

4. Richards B, Sebastian B, Sullivan H, Reyes R, D'Agostino JF, Hagerty T. Decreasing catheter-associated urinary tract infections in the neurological intensive care unit: one unit's success. Crit Care Nurse. 2017;37(3):42-8. doi: 10.4037/ ccn2017742.

5. Conway LJ, Liu J, Harris AD, Larson EL. Risk factors for bacteremia in patients with urinary catheter-associated bacteriuria. Am J Crit Care. 2016;26(1):43-52. doi: 10.4037/ ajcc2017220.

6. Chen YT, Ahmad Murad K, Ng LS, Seah JT, Park JJ, Tan TY. In vitro efficacy of six alternative antibiotics against multidrug resistant Escherichia coli and Klebsiella pneumoniae from urinary tract infections. Ann Acad Med Singapore. 2016;45(6):245-50.

7. Hirsch EB, Zucchi PC, Chen A, Raux BR, Kirby JE, McCoy C, et al. Susceptibility of multidrug-resistant gram-negative urine isolates to oral antibiotics. Antimicrob Agents Chemother. 2016;60(5):3138-40. doi: 10.1128/aac.02961-15.

8. Meletis G, Tzampaz E, Protonotariou E, Sofianou D. Emergence of Klebsiella pneumoniae carrying blaVIM and blaKPC genes. Hippokratia. 2010;14(2):139-40.

9. Cagnacci S, Gualco L, Roveta S, Mannelli S, Borgianni L, Docquier JD, et al. Bloodstream infections caused by multidrug-resistant Klebsiella pneumoniae producing the carbapenem-hydrolysing VIM-1 metallo-beta-lactamase: first Italian outbreak. J Antimicrob Chemother. 2008;61(2):296300. doi: $10.1093 / \mathrm{jac} / \mathrm{dkm} 471$.

10. Freire MP, de Oliveira Garcia D, Cury AP, Spadão F, Di Gioia TS, Francisco GR, et al. Outbreak of IMP-producing carbapenemresistant Enterobacter gergoviae among kidney transplant recipients. J Antimicrob Chemother. 2016;71(9):2577-85. doi: 10.1093/jac/dkw165.

11. Zhang F, Zhu D, Xie L, Guo X, Ni Y, Sun J. Molecular epidemiology of carbapenemase-producing Escherichia coli and the prevalence of ST131 subclone H30 in Shanghai, China. Eur J Clin Microbiol Infect Dis. 2015;34(6):1263-9. doi: 10.1007/s10096-015-2356-3.

12. Walsh TR. Emerging carbapenemases: a global perspective. Int J Antimicrob Agents. 2010;36 Suppl 3:S8-14. doi: 10.1016/ s0924-8579(10)70004-2.

13. Mathlouthi N, Al-Bayssari C, Bakour S, Rolain JM, Chouchani C. Prevalence and emergence of carbapenemases-producing gramnegative bacteria in Mediterranean basin. Crit Rev Microbiol. 2017;43(1):43-61. doi: 10.3109/1040841x.2016.1160867.

14. Agrawal P, Ghosh AN, Kumar S, Basu B, Kapila K. Prevalence of extended-spectrum beta-lactamases among Escherichia coli and Klebsiella pneumoniae isolates in a tertiary care hospital. Indian J Pathol Microbiol. 2008;51(1):139-42. doi: 10.4103/0377-4929.40428.

15. Alberti MO, Hindler JA, Humphries RM. Performance of Etest for antimicrobial susceptibility testing of Abiotrophia defectiva and Granulicatella species. J Clin Microbiol. 2016;54(8):21946. doi: 10.1128/jcm.00822-16.

16. Direkel Ş, Uzunoglu E, Uzalp Ç, Findik E, Tontak S, Ahmadli C. Determination of piperacillin/tazobactam and ticarcillin/ clavulanate susceptibilities in Pseudomonas aeruginosa isolates in hospitalised patients by E-test gradient method and comparison of results with disk diffusion tests. Clin Microbiol. 2017;6(1):273. doi: 10.4172/2327-5073.1000273.

17. Rezaeifar M, Bagheri MB, Moradi M, Rezaeifar $M$. Assessment of disk diffusion and E-test methods to determine antimicrobial activity of cefalotin and vancomycin on clinical isolates of Staphylococcus aureus. Int J Med Res Health Sci. 2016;5(11):122-6.

18. Brown DF, Brown L. Evaluation of the E test, a novel method of quantifying antimicrobial activity. J Antimicrob Chemother. 1991;27(2):185-90. doi: 10.1093/jac/27.2.185.

19. Amini M, Davati A, Golestanifard M. Frequency of nosocomial infections with antibiotic resistant strains of Acinetobacter spp. in ICU patients. Iran J Pathol. 2012;7(4):241-5.

20. Prashanth K, Badrinath S. In vitro susceptibility pattern of Acinetobacter species to commonly used cephalosporins, quinolones, and aminoglycosides. Indian J Med Microbiol. 2004;22(2):97-103.

21. Jamulitrat $S$, Thongpiyapoom $S$, Suwalak N. An outbreak of imipenem-resistant Acinetobacter baumannii at Songklanagarind hospital: the risk factors and patient prognosis. J Med Assoc Thai. 2007;90(10):2181-91.

22. Malone KM, Gordon SV. Antibiotic methylation: a new mechanism of antimicrobial resistance. Trends Microbiol. 2016;24(10):771-2. doi: 10.1016/j.tim.2016.08.003.

23. Ranjbar R, Sadeghifard N, Ahmadi A, Izadi M, Zaeimi-Yazdi J, Ghasemi A, et al. Antimicrobial susceptibility and AP-PCR typing of Acinetobacter spp. strains. Iran J Public Health. 2007;36(4):50-6.

24. Manoharan A, Pai R, Shankar V, Thomas K, Lalitha MK. Comparison of disc diffusion \& $E$ test methods with agar dilution for antimicrobial susceptibility testing of Haemophilus influenzae. Indian J Med Res. 2003;117:81-7.

25. Nobari S, Shahcheraghi F, Rahmati Ghezelgeh F, Valizadeh B. Molecular characterization of carbapenem-resistant strains of Klebsiella pneumoniae isolated from Iranian patients: first identification of blaKPC gene in Iran. Microb Drug Resist. 2014;20(4):285-93. doi: 10.1089/mdr.2013.0074.

26. Simhon A, Rahav G, Shazberg G, Block C, Bercovier H, Shapiro M. Acinetobacter baumannii at a tertiary-care teaching hospital in Jerusalem, Israel. J Clin Microbiol. 2001;39(1):389-91. doi: 10.1128/jcm.39.1.389-391.2001.

27. Bora A, Sanjana R, Jha BK, Mahaseth SN, Pokharel K. Incidence of metallo-beta-lactamase producing clinical isolates of Escherichia coli and Klebsiella pneumoniae in central Nepal. BMC Res Notes. 2014;7:557. doi: 10.1186/1756-0500-7-557.

28. Moayednia R, Shokri D, Mobasherizadeh S, Baradaran A, Fatemi SM, Merrikhi A. Frequency assessment of beta-lactamase enzymes in Escherichia coli and Klebsiella isolates in patients with urinary tract infection. J Res Med Sci. 2014;19(Suppl 1):S41-5.

29. Shahcheraghi F, Nobari S, Rahmati Ghezelgeh F, Nasiri S, Owlia P, Nikbin VS, et al. First report of New Delhi metallo-betalactamase-1-producing Klebsiella pneumoniae in Iran. Microb Drug Resist. 2013;19(1):30-6. doi: 10.1089/mdr.2012.0078.

30. Adwan G, Bourinee H, Othman S. Prevalence of metallo-betalactamases producing Escherichia coli isolated from North of Palestine. Journal of Microbiology and Antimicrobial Agents. 2016;2(1):9-15.

31. Kanchanadevi $P$, Sekaran SC. Importance of EDTA in the detection of metallo beta lactamase from imipenem resistant gram negative bacilli. Int J Curr Microbiol Appl Sci. 2016;5(11):702-6. 